\title{
GPU Implementation of the Electric Power System Model for Real-Time Simulation of Electromagnetic Transients
}

\author{
Slawomir Cieslik \\ Electrical Engineering Institute \\ University of Technology and Life Sciences \\ Bydgoszcz, Poland \\ e-mail: slavcies@utp.edu.pl
}

\begin{abstract}
This paper presents decomposition method of mathematical model of electric power system and implementation its in digital system with GPU. The electric power system model can be used for real-time simulation of electromagnetic transients. The article presents the results of experimental research of calculation time for two different digital systems using both the CPU and GPU.
\end{abstract}

Keywords-electromagnetic transients; real-time simulation; parallel processing; GPU; thread computing; CUDA

\section{INTRODUCTION}

Simulation of electromagnetic transients in electric power systems is one of the basic research methods. Complicated algorithms of digital controllers used in automatic regulation of electric power systems often hinders reliable modeling of its electromagnetic transients (especially if neural networks and genetic algorithms are used). One of the ways of performing a reliable simulation of electric power systems with digital regulators is using of a real-time electric power system simulator, which allows to connect a real controller (in which case there is no need to define its characteristics, as it is interacting with the simulator). Such a simulator needs to generate results with minimal errors as well as promptly calculate the data in order to enable the communication with the environment (i.e. the real controller).

The mathematical model of the electric power system is an integral part of the simulator. One of the ways of implementing the mathematical models for the digital realtime power system simulator is their decomposition through various methods. Therefore, it is appropriate to research on mathematical models of electric power systems in the aspect of results accuracy and calculation time.

This article describes the method of mathematical model decomposition based on the partition of the modeled power system into structural elements represents as electric multipoles $[1,2]$. The problem of improving the accuracy of the results has been discussed in author's previous work [1], in which there is shown an example of implementing various algorithms for integration of differential equations for certain groups of structural elements.

The issue of calculation time is also considered in this article. In order to shortening solving time in PC-based digital simulators GPUs are used $[3,4,5]$.

The CUDA architecture enables a relatively easy implementation of the decomposed mathematical model and parallel calculations in separated blocks which are responsible for respective structural elements.

This article presents the results of a research on electromagnetic transients in an exemplary electric power system with the use of two different digital systems: CPU Intel Core i7 970 with GPU NVIDIA GE Force GTX 650 and CPU Intel Core i7 2630 QM with GPU NVIDIA GE Force GT 540M.

\section{The Method Of Model Decompositon}

The method of mathematical modeling with the use of electric multipoles is one of many methods [6,7] of mathematical modeling of electric power systems, but it is one of the few that allows the model decomposition, so that the calculations are execute in parallel.

The concept of using electric multipoles is successfully applied in mathematical modeling of complex electromechanical and electric power systems.

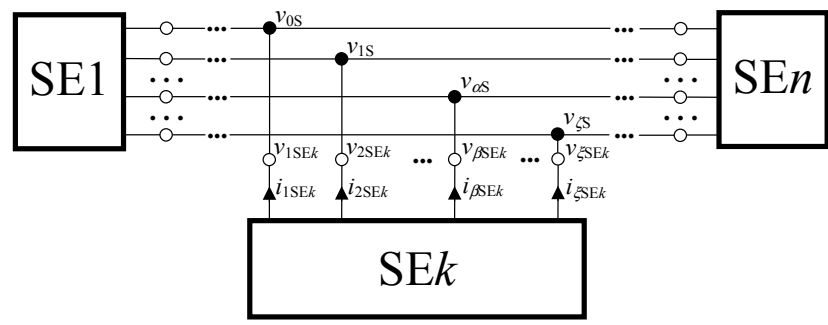

Figure 1. The equivalent scheme of the modeled electric power system.

The electric power system shown on Figure 1. is considered as a electric connection of $n$ structural elements (SE). Every $k$-th structural element SE $k$ is represented in general case by electric multipole with matrices:

$$
v_{\mathrm{SE} k}=\left[\begin{array}{llllll}
v_{1 S \mathrm{E} k} & v_{2 S \mathrm{~S} k} & \ldots & v_{\beta \mathrm{SE} k} & \ldots & v_{\xi \mathrm{SE} k}
\end{array}\right]^{\mathrm{T}},
$$

- electric potentials of the SE $k$ external nodes,

$$
\boldsymbol{i}_{\mathrm{SE} k}=\left[\begin{array}{llllll}
i_{1 S \mathrm{SE} k} & i_{2 \mathrm{SE} k} & \ldots & i_{\beta \mathrm{SE} k} & \ldots & i_{\xi \mathrm{SE} k}
\end{array}\right]^{\mathrm{T}},
$$

- external branch currents of SE $k$. 
The mathematical representation of each SE $k$ has to be carried out in such a way that we can provide an external equation in the form as follows

$$
\boldsymbol{i}_{\mathrm{SE} k}+\boldsymbol{A}_{\mathrm{SE} k} \boldsymbol{v}_{\mathrm{SE} k}+\boldsymbol{B}_{\mathrm{SE} k}=\mathbf{0}
$$

There is a square matrix $\boldsymbol{A}_{\mathrm{SE} k}$ with dimensions equal $\xi \times \xi$ in the (3) equation. Also we can find there the $\xi$ element matrix $\boldsymbol{B}_{\mathrm{SEk} k}$. The elements of the mentioned matrix are defined by structural element parameters and internal physical variables associated with the SE $k$.

The external branches of structural elements are connected at $(\zeta+1)$ nodes of electric power system ( $0 \mathrm{~S}$, $1 \mathrm{~S}, \ldots, \alpha \mathrm{S}, \ldots, \zeta \mathrm{S})$. The electric potential of one of them is zero $\left(v_{0 \mathrm{~S}}=0\right)$, therefore for the remaining $\zeta$-th independent nodes we can define the following matrix

$$
\boldsymbol{v}_{\mathrm{S}}=\left[\begin{array}{lllll}
v_{1 \mathrm{~S}} & \ldots & v_{\alpha \mathrm{S}} & \ldots & v_{\zeta \mathrm{S}}
\end{array}\right]^{\mathrm{T}},
$$

called by electric potential nodes matrix of the electric power system.

Relationships between electric potentials of external nodes of structural elements and electric potentials of nodes of the electric power system are expressed using the transposed of an incidence matrix $\boldsymbol{P}_{k}$ for each $k$-th element, as follows

$$
\boldsymbol{v}_{\mathrm{SE} k}=\boldsymbol{P}_{k}^{\mathrm{T}} \boldsymbol{v}_{\mathrm{S}}
$$

$\boldsymbol{P}_{k}$ matrices appearing in the (5) equation are constant for a given SE. The number of rows of the given matrix is equal to the number of independent nodes of the electric power system, and the number of columns is equal to the number of external branches of structural element. The matrix element on the intersection of the row and the column is equal to one only if the corresponding to the number of the node line number is connected to a branch of the multipole with a number corresponding to the number of the column. In other cases, the elements of this matrix are equal to zero.

Using the 1st Kirchhoff's law for all independent electric power system nodes we receive the equation

$$
\sum_{j=1}^{n} \boldsymbol{P}_{j} \mathbf{i}_{\mathrm{SE} j}=\mathbf{0}
$$

where the external branch currents of the structural elements were inserted and designated from external equations of multipoles (3). Regarding to the equation (5) received the following nodal equation

$$
A_{\mathrm{S}} v_{\mathrm{S}}+B_{\mathrm{S}}=\mathbf{0}
$$

$$
\boldsymbol{A}_{\mathrm{S}}=\sum_{j=1}^{n} \boldsymbol{P}_{j} \boldsymbol{A}_{\mathrm{SEj}} \boldsymbol{P}_{j}^{\mathrm{T}} \text { and } \boldsymbol{B}_{\mathrm{S}}=\sum_{j=1}^{n} \boldsymbol{P}_{j} \boldsymbol{B}_{\mathrm{SE} j} .
$$

Mathematical modelling of electromagnetic transients in electric power systems is done according to the algorithm shown in Figure 2.

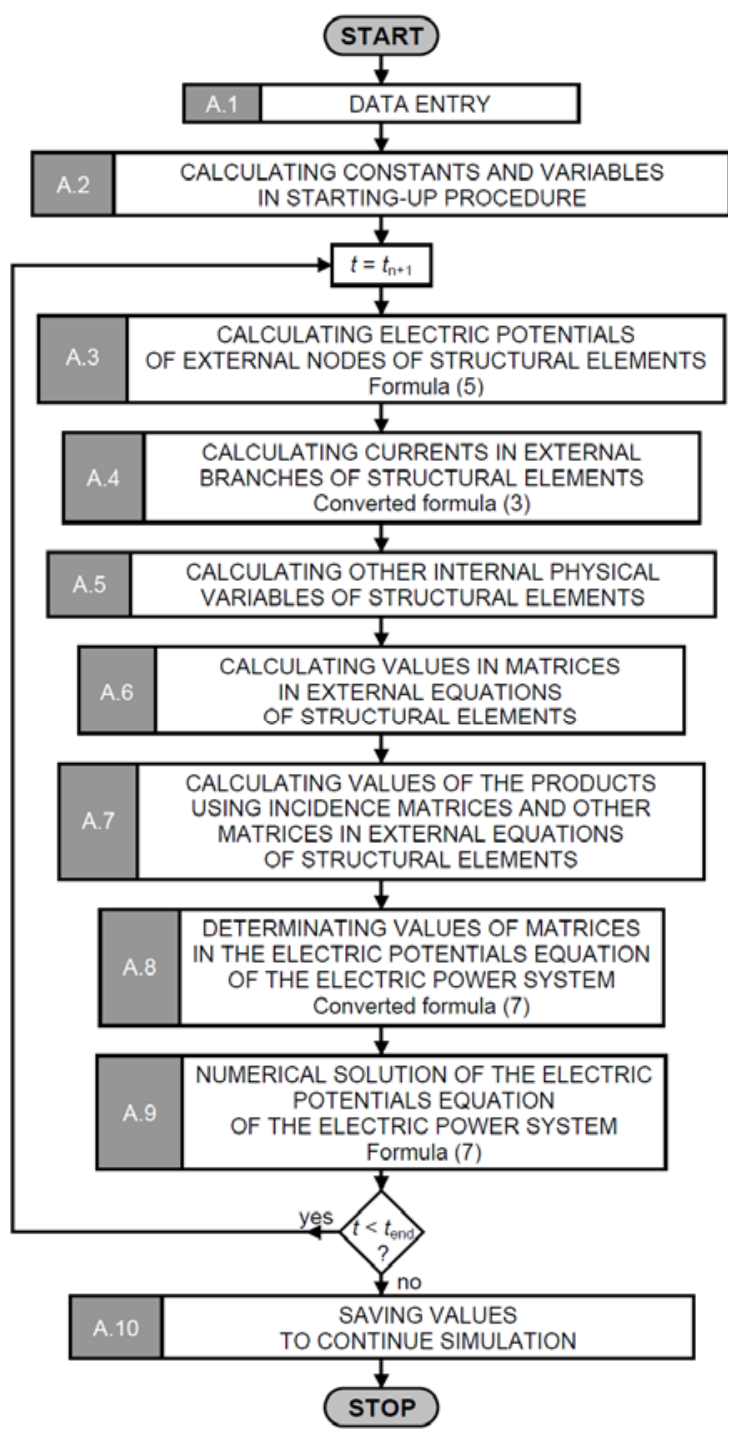

Figure 2. Mathematical modelling algorithm.

The goal is that the tasks inside the loop shown on Figure 2 should perform in parallel. There are examples of using certain numerical methods of solving systems of equations using the GPU in the literature [8,9]. In this article there is a particular emphasis the possibility of parallel tasks and calculations A.3. - A.8., which is associated with decomposing the mathematical model.

where: 


\section{MAthematical Model of 3-Phase Structural ELEMENT}

Parallel numerical calculations in the CUDA environment are done with the best performance, only if in each block and each thread exactly the same computational tasks are implemented, except inserting different data.

Decomposition mathematical model of an electric power system, which is to be implemented in the CUDA architecture, apply the most consistent three-phase model of the structural element, whose equivalent scheme is shown in Figure 3. Also there is a list of the parameters of a mathematical model of this structural element in the Table I.

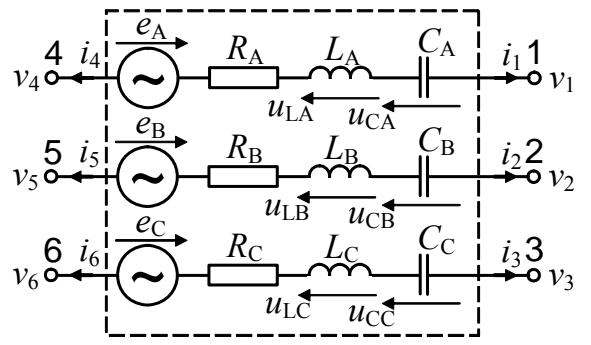

Figure 3. The equivalent scheme of three-phase structural element.

TABLE I. PARAMETERS OF THE MATHEMATICAL MODEL OF THE STRUCTURAL ELEMENT

\begin{tabular}{|c|c|c|}
\hline No & Symbol & Parameter description \\
\hline 1 & $E_{0 \zeta}$ & $\zeta$-phase voltage offset $e_{\zeta}(t)[\mathrm{V}]$ \\
\hline 2 & $E_{\mathrm{m} \zeta \lambda}$ & $\zeta$-harmonics voltage amplitude $e_{\zeta}(t)[\mathrm{V}]$ \\
\hline 3 & $\psi_{\mathrm{e} \zeta \lambda}$ & $\zeta$-phase, $\lambda$-harmonics of voltage $e_{\zeta}(t)[\mathrm{rad}]$ \\
\hline 4 & $f$ & Frequency ${ }^{(1)}$ of voltage sources $e_{\zeta}(t)[\mathrm{Hz}]$ \\
\hline 5 & $R_{\zeta}$ & $\zeta$-th phase element resistance $[\Omega]$ \\
\hline 6 & $L_{\zeta}$ & $\zeta$-th phase element inductance $[\mathrm{H}]$ \\
\hline 7 & $C_{\zeta}$ & $\zeta$-th phase element capacitance $[\mathrm{F}]$ \\
\hline (1) $\begin{array}{c}\text { In the general case each phase source voltages frequencies are } \\
\text { different. }\end{array}$
\end{tabular}

The mathematical model of the structural element based on discrete mathematical models of inductance $L$ and capacity $C$ was proposed in $\lceil 10\rceil$.

After use trapezoidal rule the following dependencies for matrix values are derived $\boldsymbol{A}_{\mathrm{SE}}=\left[\begin{array}{cc}\boldsymbol{a}_{\mathrm{SE}} & -\boldsymbol{a}_{\mathrm{SE}} \\ -\boldsymbol{a}_{\mathrm{SE}} & \boldsymbol{a}_{\mathrm{SE}}\end{array}\right]$, where $\boldsymbol{a}_{\mathrm{SE}}=\operatorname{diag}\left(\alpha_{\mathrm{ASE}}, \alpha_{\mathrm{BSE}}, \alpha_{\mathrm{CSE}}\right)$, and for the vector $\boldsymbol{B}_{\mathrm{SE}}=\left[\begin{array}{c}\boldsymbol{b}_{\mathrm{SE}} \\ -\boldsymbol{b}_{\mathrm{SE}}\end{array}\right]$, where $\boldsymbol{b}_{\mathrm{SE}}=\left[\begin{array}{lll}\beta_{\mathrm{ASE}} & \beta_{\mathrm{BSE}} & \beta_{\mathrm{CSE}}\end{array}\right]^{\mathrm{T}}$ in external equation (3):

$$
\alpha_{\zeta \mathrm{SE}}=\left(R_{\zeta \mathrm{SE}}+\xi^{-1} L_{\zeta \mathrm{SE}}+\xi C_{\zeta \mathrm{SE}}^{-1}\right)^{-1}
$$

$$
\begin{aligned}
\beta_{\zeta \mathrm{SE}}= & \alpha_{\zeta \mathrm{SE}}\left(-e_{\zeta \mathrm{SE}}\left(t_{\mathrm{n}+1}\right)-u_{\mathrm{L} \zeta \zeta \mathrm{S}}\left(t_{\mathrm{n}}\right)+\right. \\
& \left.-\left(L_{\zeta \mathrm{SE}} \xi^{-1}-\xi C_{\zeta \mathrm{SE}}^{-1}\right) i_{k S \mathrm{SE}}\left(t_{\mathrm{n}}\right)+u_{\mathrm{C} \zeta \zeta \mathrm{S}}\left(t_{\mathrm{n}}\right)\right),
\end{aligned}
$$

where $\xi=0,5 h$

Capacitor voltages are equal

$u_{\mathrm{C} \zeta \mathrm{E}}\left(t_{\mathrm{n}+1}\right)=\xi C_{\zeta \mathrm{ES}}^{-1}\left(i_{k \mathrm{ES}}\left(t_{\mathrm{n}+1}\right)+i_{k \mathrm{ES}}\left(t_{\mathrm{n}}\right)\right)+u_{\mathrm{C} \zeta \zeta \mathrm{E}}\left(t_{\mathrm{n}}\right)$,

Inductor voltages are equal

$$
u_{\mathrm{L} \zeta \zeta \mathrm{E}}\left(t_{\mathrm{n}+1}\right)=L \xi^{-1}\left(i_{k \mathrm{ES}}\left(t_{\mathrm{n}+1}\right)-i_{k \mathrm{ES}}\left(t_{\mathrm{n}}\right)\right)-u_{\mathrm{L} \zeta \zeta \mathrm{E}}\left(t_{\mathrm{n}}\right) .
$$

\section{Description Of Modeled Electric Power SYSTEM}

Figure 2. shows a $15 \mathrm{kV}$ electric power system schematic diagram, meanwhile Figure 3 . is treated as the equivalent scheme. The system consists of 25 (SE1-SE25) structural elements.

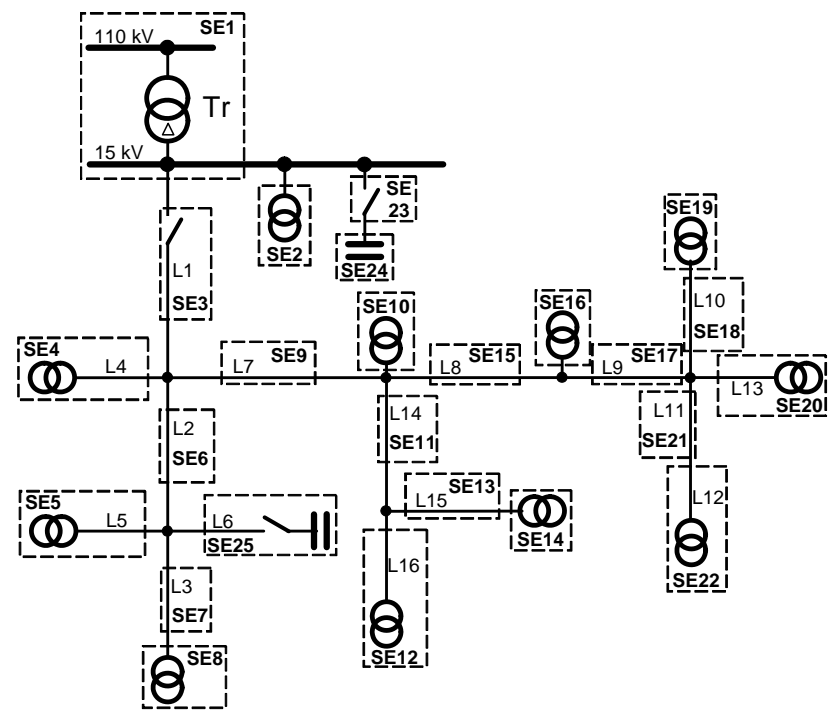

Figure 4. Schematic diagram of modeled electric power system [1]. 


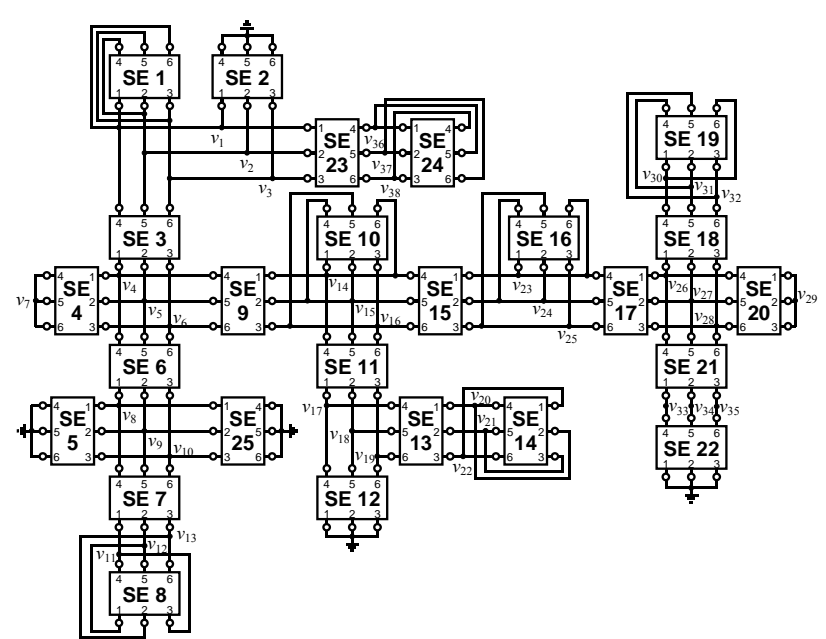

Figure 5. Equivalent scheme of modeled electric power system [1].

In this case, most of the structural elements of the model corresponds to exactly one element of the real system, e.g. SE9 corresponds to the amount the line marked as L7. There are also several cases in which the structural element of the model corresponds to the group of elements of the real system, for example SE25 contains electric line segment marked as L6, a power switch and a capacitors bank. The partition of the electric power system structure (decomposition) for structural elements is a very important problem in terms of calculation time (The problem of improving the accuracy of the results has been discussed in author's previous work [1]). On the equivalent scheme, each structural element is represented as an electric six-pole, as shown in Figure 3.

\section{EXPERIMENTAL RESULTS AND CONCLUSIONS}

Figures 6-8 shows results (computation times) of experimental computational tests carried out on two digital systems. In 2-nd block system of equations is solved, and in 1-st block A.3-A.8 tasks are executed as showed on algorithm (Figure 2).

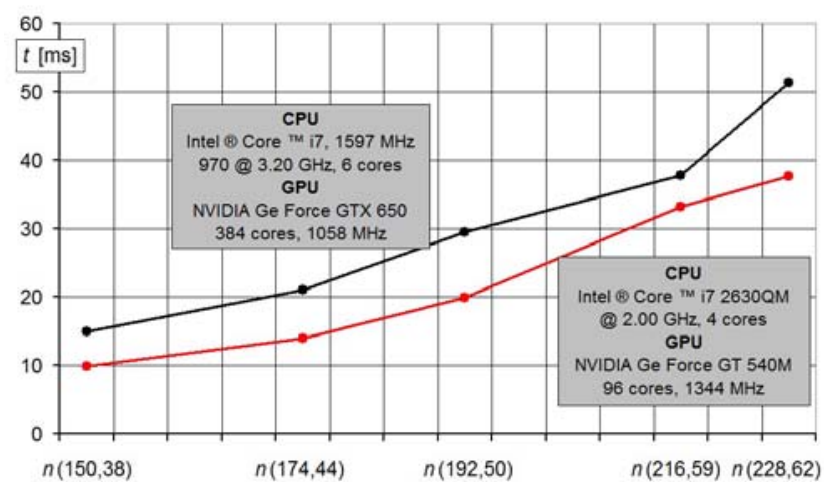

Figure 6. Sequential computation total execution times measured on two digital systems.

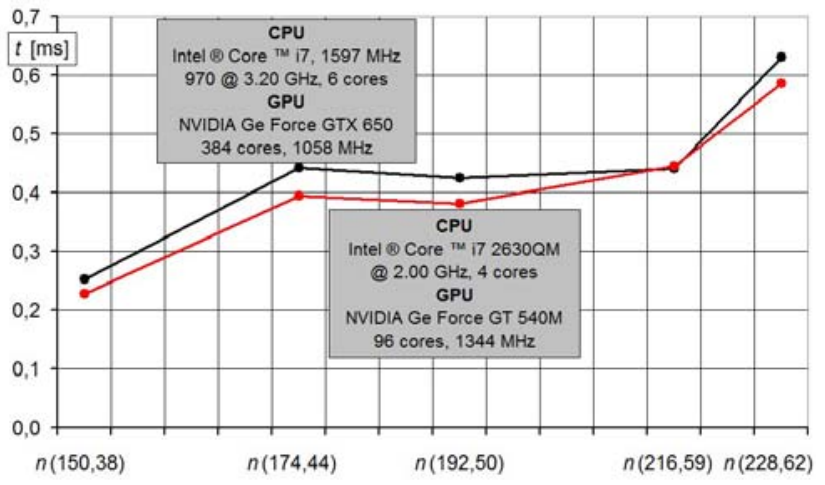

Figure 7. Parallel computation total execution times measured on two digital systems.

The size of computational task is presented on $\mathrm{X}$-axis, $n(150,38)$ means that task consists of 150 -variables in the model of electric power system solved on each integration step and 38-nodes of electric power system - case shown on Figure 4. Next four cases apply to more complex computational problems: $n(174,44), n(192,50), n(216,59)$ and $n(228,62)$.

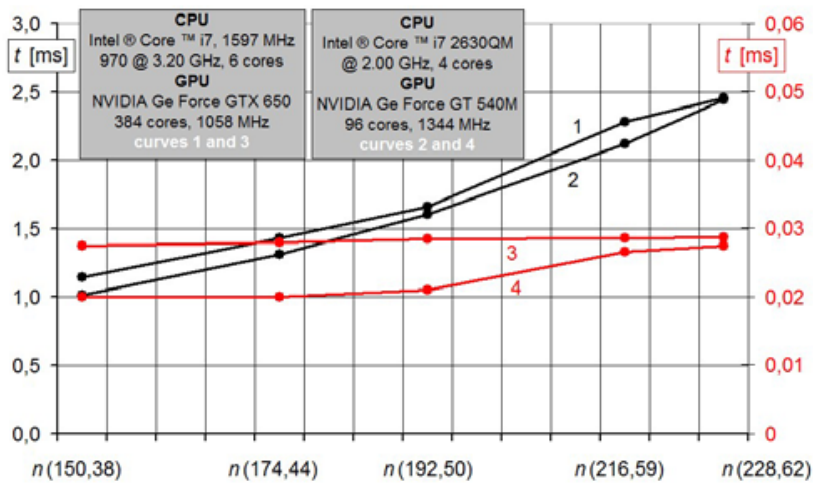

Figure 8. 1-st block arallel computation execution times measured on two digital systems.

Please pay attention that parallel computation time of 1 -st block is approximately constant (it's true only if structural elements number is less than GPU-cores number).

Described results showed, that computation time on GPU-used system with more cores (384) was longer than on less cores (96). The reason is $27 \%$ higher system clock frequency in $2^{\text {nd }}$ case (1344 MHz vs. $1058 \mathrm{MHz}$ ).

\section{REFERENCES}

[1] S. Cieslik, "Decomposition of Mathematcal Models of Power Systems for Real-Time Digital Simulators in Aspect of Calculation Accuracy", Rynek Energii, vol. 1(98), Feb. 2012, pp. 47-53, ISSN 1425-5960.

[2] S. Cieslik, "Digital Simulators as an Assessment Tool of the Impact of Distributed Generation on Power Grid Infrastructure", Electrical Review, vol. 2010. No. 8, Aug. 2010, pp. 253-260, ISSN 0033-2097. 
[3] M. Drechny, "Possibility of Parallel Computing Application in Power Engineering", Rynek Energii, vol. 4(101), Aug. 2012, pp. 66-70, ISSN 1425-5960.

[4] L. Murray, "GPU Acceleration of Runge-Kutta Integrators", IEEE Transactiona on Parallel and Distributed Systems, vol. 23. No. 1, Jan. 2012, pp. 94-101, 1045-9219/12.

[5] J.K. Debnath, Wai-Keung Fung, A.M. Gole and S. Filizadeh, "Simulation of Large-Scale Electrical Power Networks on Graphics Processing Units", Proc. IEEE Electrical Power and Energy Conference, 2011, pp. 199-204, 978-1-4577-0404-8/11.

[6] P. Zang, J.R. Martí and H.W. Dommel, "Network Partitioning for Real-Time power System Simulation", International Conference on Power Systems Transients (IPST’05), Montreal, Canada, June 2005, Paper No. IPST05-177.

[7] J.R. Martí, L.R. Linares, J. Calviño and H.W. Dommel, "OVNI: An Object Approach to Real-Time Power Simulators", Proc. of the International Conference on Power System Technology, Beijing, China, Aug. 1998, pp. 1-5.

[8] G. Amador and A. Gomes, "Linear Solvers for Stable Fluids: GPU vs CPU”, Proc. of the $17^{\text {th }}$ Encontro Portugeês de Computação Gráfica (EPCG 09), 2009, pp. 145-153.

[9] H. Courtecuisse and J. Allard, "Parallel Dense Gauss-Seidel Algorithm on Many-Core Processors", Proc. of $11^{\text {th }}$ IEEE International Conference on High Performance Computing and Communications, June 2009, pp. 139-147.

[10] H.W. Dommel, "Digital computer solution of electromagnetic transients in single- and multiphase networks", IEEE Transactions on Power Apparatus and Systems, Vol. PAS-88, 1969, pp. 388-399. 